\title{
DYNAMIC ROUTING AND ADMISSION CONTROL FOR VIRTUAL CIRCUIT NETWORKS ${ }^{1}$
}

\author{
by
}

\author{
A. A. Economides ${ }^{2}$, P. A. Ioannou ${ }^{3}$ and J. A. Silvester ${ }^{4}$
}

\begin{abstract}
The dynamic joint routing and admission control problem in multiple class multiple source-destination virtual circuit networks is considered.

A nonlinear dynamic queueing model for virtual circuit networks that considers the dynamic interaction among the virtual circuit and packet processes is introduced. Then a multi-objective cost function of rejecting \& maintaining virtual circuits, as well as of delaying \& servicing packets is defined.

The combined problem is formulated as an optimal control problem. Necessary optimality conditions are provided by Pontryagin's maximum principle. Sufficient optimality conditions based on the convexity of the Hamiltonian function are also given. For the finite horizon, the optimal controls can be found after numerically solving a Two-Point Boundary-Value Problem. For the long-run stationary equilibrium, the state-dependent routing and admission controls are derived.
\end{abstract}

Key words: dynamic admission control, dynamic routing, multi-objective, optimal control, virtual circuit networks.

\footnotetext{
${ }^{1}$ This work was supported by the National Science Foundation under Grant DMC-8452002 together with matching funds from AT\&T Information Systems.

${ }^{2}$ University of Macedonia, Thessaloniki 54006, GREECE

${ }^{3}$ University of Southern California, Los Angeles, CA 90089-2562, USA

${ }^{4}$ University of Southern California, Los Angeles, CA 90089-2562, USA
}

Correspondence should be directed to A. A. Economides, University of Macedonia, Thessaloniki 54006, GREECE, Tel \# 3031-891799, Fax \# 3031-834070, e-mail: economid@macedonia.uom.gr 


\section{INTRODUCTION}

Most existing networks (Codex, Euronet, SNA, Telenet, Transpac, Tymnet, etc.) as well as proposals for future high speed network architectures employ virtual circuit switching. For each call (virtual circuit, or virtual channel, or virtual connection, or virtual route, or session, or transaction, etc.), a single path is set up from source to destination and all entities (bursts, packets, cells, etc.) that belong to this call follow this path.

Two of the most important algorithms for efficient virtual circuit network control are routing and admission control. Routing decides which route the virtual circuit will follow from source to destination. Admission control prevents network overload by controlling the virtual circuit traffic entering the network. Routing and admission control are strongly related problems and each affects the other. For a more accurate model and better network performance, both problems should be modeled and solved simultaneously. Such an approach however may increase the modeling and optimization complexity. Previous studies on virtual circuit network control usually concentrate on the routing problem. In this paper, we consider the combined virtual circuit dynamic routing and admission control problem.

In a real virtual circuit network, the network state is continuously changing due to real time traffic fluctuations. Therefore, the routing and admission control decisions should depend on the current network state, for example the current network topology, the current number of packets \& virtual circuits, the current virtual circuit \& packet arrival rates, the current service requirements, the current link error rates etc.

Such a stochastic problem is extremely difficult even under Markovian assumptions. Since the decisions should depend on the current network state, we must find the transient solution of the corresponding Markov Chain with time dependent external arrival and service rates (recall the nasty expressions for the transient analysis of a simple $M / M / 1$ queue [1]). Finally, even if we solve the corresponding Markov Chain and find statedependent controls that depend on the current network state, it is impossible to know the current network state at all network resources, every moment. The needed time for the network state observations to be transferred from one network point to another is a random variable that also depends on the network state and during this time the network state has already changed. Furthermore, it is also difficult to obtain accurate estimates of the instantaneous rates. So, the network state information is always obsolete and inaccurate. Therefore attacking the stochastic problem directly would be difficult.

Also, in real network control implementations, the average rather than the instantaneous measures of the network state are used due to the following reasons: 1) wide variability of the instantaneous network state values, 2) obsolete network state information, 
due to transfer delay, 3) periodic implementation of the network control, 4) communication overhead in transferring the instantaneous network state information, and 5) computation overhead in calculation for an exact network optimization.

Previous studies on the dynamic virtual circuit routing problem $[2,3,4,5,6]$ do not explicitly consider the dynamic interaction between the virtual circuit and the packet processes. Also, the admission control problem has not been considered jointly with the routing problem, although these problems are strongly related. In this paper, we extend our results $[7,8]$ on modeling virtual circuit networks and deriving the optimal routing and admission controls. The network dynamics occur at two different time scales. The virtual circuit process evolves at the slower time scale and is used in the evolution of the packet process that occurs at the faster time scale. In section 2, we introduce a nonlinear dynamic queueing model that describes the dynamic interaction among the virtual circuit and packet processes. In section 3, we set up a multi-objective cost function of rejecting and maintaining virtual circuits, as well as of the packet delay and throughput. Then we formulate the combined routing and admission control problem as an optimal control problem. Pontryagin's maximum principle provides necessary optimality conditions that are also sufficient. For the finite horizon problem, a Two-Point Boundary-Value Problem must be solved numerically. For the long-run stationary equilibrium, in section 4, we derive state-dependent virtual circuit routing and admission controls. In $[9,10]$, we show via simulation the superiority of this state-dependent virtual circuit routing to the shortestqueue routing.

\section{VIRTUAL CIRCUIT NETWORK MODEL}

Consider an arbitrary network topology with multiple classes of virtual circuit traffic between multiple source-destination pairs. Instead of introducing an extra notational index for each class of virtual circuits, we can consider each class $c$ of virtual circuits between a source-destination pair $[s d]$ as being established between a fictitious $\left[s_{c} d_{c}\right]$ pair, where physically $s_{c}=s$ and $d_{c}=d, \forall c$. The queueing models that we introduce in this section can handle this substitution. Note also that one extreme case is to consider each virtual circuit as a different class. Another extreme case is to consider all virtual circuits as belonging to the same class. Also, in contemporary networks, the nodal processing delays are negligible compared to the transmission and propagation delays and therefore they were ignored in network optimization and control procedures. However, in future high speed networks, the transmission delays will be very short and comparable to the nodal processing delays. Therefore, packets will be queued not only in front of the links but also in front of the nodes. However, instead of introducing extra variables to describe the state of each node, we can consider each node $i$ as a link $i_{1} i_{2}$. So, in the following 
analysis, the word "link" may mean physically either a link or a node.

Virtual circuits arrive at a source node $s$ (according to a Poisson distribution) destined to a destination node $d$ with rate $\gamma_{[s d]}(t) \geq 0$. For admission control reasons, a fraction $\phi_{o[s d]}(t) \in[0,1]$ of these externally arriving $[s d]$ virtual circuits is rejected, while the remaining virtual circuits are accepted into the network. A fraction $\phi_{\pi[s d]}(t) \in[0,1]$ of the externally arriving $[s d]$ virtual circuits are routed from node $s$ to its destination node $d$ through path $\pi[s d]$, where $\phi_{o[s d]}(t)+\sum_{\pi[s d]} \phi_{\pi[s d]}(t)=1$. Then the rejected $[s d]$ virtual circuit flow at the source node $s$ is $\gamma_{[s d]}(t) * \phi_{o[s d]}(t)$ and the $[s d]$ virtual circuit flow on path $\pi[s d]$ is $\gamma_{[s d]}(t) * \phi_{\pi[s d]}(t)$. The above procedure happens for every source-destination pair in the network. Therefore the $[s d]$ virtual circuit flow on link $i j$ is the sum of the $[s d]$ virtual circuit flows of all paths traversing this link, i.e. $\sum_{\pi[s d]} \gamma_{[s d]}(t) * \phi_{\pi[s d]}(t) * 1_{i j \in \pi[s d]}(t)$, where $1_{i j \in \pi[s d]}(t)$ means that at time $t$ link $i j$ belongs to path $\pi[s d]$, or that path $\pi[s d]$ traverses link $i j$.

Packets generated in each $[s d]$ virtual circuit arrive according to a Poisson distribution and the number of packets per virtual circuit is geometrically distributed. Then the $[s d]$ virtual circuit duration (lifetime) is exponentially distributed. Let $1 / \delta_{[s d]}(t) \geq 0$ be the mean virtual circuit duration. So, we can model every link $i j$ for the $[s d]$ virtual circuit process as an $M / M / \infty$ queue with arrival rate $\sum_{\pi[s d]} \gamma_{[s d]}(t) * \phi_{\pi[s d]}(t) * 1_{i j \in \pi[s d]}(t)$ and mean service time $1 / \delta_{[s d]}(t)$. We note that thousands of virtual circuits can coexist on a link (well within today's technology capabilities) [11].

Subsequently, we will introduce a state space approach to model the dynamic evolution of the virtual circuit processes. The expected number of $[s d]$ virtual circuits on link $i j$ at time $t, V_{i j[s d]}(t) \geq 0$, increases during $\Delta t$ by the expected number of $[s d]$ virtual circuits that arrive during this period, $\sum_{\pi[s d]} \gamma_{[s d]}(t) * \phi_{\pi[s d]}(t) * 1_{i j \in \pi[s d]}(t) * \Delta t$, minus the expected number of $[s d]$ virtual circuits that depart during this period, $\delta_{[s d]}(t) * V_{i j[s d]}(t) * \Delta t$. So, the $[s d]$ virtual circuit process at link $i j$ is described by

$$
\begin{aligned}
& V_{i j[s d]}(t+\Delta t)=V_{i j[s d]}(t)+\sum_{\pi[s d]} \gamma_{[s d]}(t) * \phi_{\pi[s d]}(t) * 1_{i j \in \pi[s d]}(t) * \Delta t-\delta_{[s d]}(t) * V_{i j[s d]}(t) * \Delta t \\
& \quad \forall i j \quad \forall[s d]
\end{aligned}
$$

The expected number of $[s d]$ virtual circuits on every link $i j$ at time $t, V_{i j[s d]}(t)$, is a 
continuous function of time, so let us define

$$
\dot{V}_{i j[s d]}(t)=\lim _{\Delta t \rightarrow 0} \frac{V_{i j[s d]}(t+\Delta t)-V_{i j[s d]}(t)}{\Delta t} \quad \forall i j \quad \forall[s d]
$$

Therefore the $[s d]$ virtual circuit process on link $i j$ at time $t$ is described by

$$
\dot{V}_{i j[s d]}(t)=\sum_{\pi[s d]} \gamma_{[s d]}(t) * \phi_{\pi[s d]}(t) * 1_{i j \in \pi[s d]}(t)-\delta_{[s d]}(t) * V_{i j[s d]}(t) \quad \forall i j \quad \forall[s d]
$$

Next, we describe the evolution of the packet process into the network. Let $r_{[s d]}(t) \geq 0$ be the packet arrival rate per $[s d]$ virtual circuit at time $t$ (Poisson distribution). If there are $V_{i j[s d]}(t)[s d]$ virtual circuits on link $i j$ at time $t$, then the total $[s d]$ packet arrival rate to link $i j$ is $r_{[s d]}(t) * V_{i j[s d]}(t)$, since all packets belonging to a virtual circuit are transmitted through the same link. Note also, that the mean number of packets generated in each $[s d]$ virtual circuit is $r_{[s d]}(t) / \delta_{[s d]}(t) \geq 0$.

Let the packet service requirement be exponentially distributed with mean $1 / \mu>0$ and the service rate at link $i j$ be $C_{i j}>0$. Then the mean packet service time at link $i j$ is $1 / \mu_{i j}=1 /\left(\mu * C_{i j}\right)$. Packets are serviced according to first-come-first-served or processor sharing scheduling. Katevenis [11] and Morgan [12] preallocate buffer space to each virtual circuit in every node and multiplex packets from different (thousands) virtual circuits using round-robin scheduling. So, for the $[s d]$ packet process, we model each link $i j$ either as an $M / M / 1$ or as a Processor Sharing queue, with packet arrival rate $r_{[s d]}(t) * V_{i j[s d]}(t)$ and mean service time $1 / \mu_{i j}(t)$. Note, that for the Processor Sharing discipline, the packet service requirement may be generally distributed and packets from different classes of virtual circuits may have different mean service requirements.

Let $N_{i j[s d]}(t) \geq 0$ be the expected number of $[s d]$ packets at link $i j$ at time $t$ and $\mathbf{N}_{i j}(t)=\left[\ldots N_{i j[s d]}(t) \ldots\right]^{T}$ be the vector of the expected number of packets on link $i j$ for all source-destination processes. Let $\rho_{i j[s d]}\left(\mathbf{N}_{i j}(t)\right)$ be the probability that there is at least an $[s d]$ packet at link $i j$ (either in queue or in transmission) at time $t$ (call this probability: "instantaneous utilization for link $i j$ for the $[s d]$ traffic"), such that the $[s d]$ packet departure rate from link $i j$ at time $t$ is $\mu_{i j}(t) * \rho_{i j[s d]}\left(\mathbf{N}_{i j}(t)\right)$.

Then the expected number of $[s d]$ packets at link $i j$ at time $t, N_{i j[s d]}(t)$, increases during $\Delta t$ by the expected number of $[s d]$ packets that arrive during this period, $r_{[s d]}(t) *$ $V_{i j[s d]}(t) * \Delta t$, minus the expected number of $[s d]$ packets that depart during this period, $\mu_{i j}(t) * \rho_{i j[s d]}\left(\mathbf{N}_{i j}(t)\right)$. Since, the link utilization $\rho_{i j[s d]}\left(\mathbf{N}_{i j}(t)\right)$, is a nonlinear function of 
the number of packets at link $i j, \mathbf{N}_{i j}(t)$, the $[s d]$ packet process at link $i j$ is described by the following nonlinear dynamic model

$$
\begin{aligned}
& N_{i j[s d]}(t+\Delta t)=N_{i j[s d]}(t)+r_{[s d]}(t) * V_{i j[s d]}(t) * \Delta t-\mu_{i j}(t) * \rho_{i j[s d]}(t)\left(\mathbf{N}_{i j}(t)\right) * \Delta t \\
& \forall i j \quad \forall[s d]
\end{aligned}
$$

The expected number of $[s d]$ packets at link $i j$ at time $t, N_{i j[s d]}(t)$, is a continuous function of time. So, let us define

$$
\dot{N}_{i j[s d]}(t)=\lim _{\Delta t \rightarrow 0} \frac{N_{i j[s d]}(t+\Delta t)-N_{i j[s d]}(t)}{\Delta t} \quad \forall i j \quad \forall[s d]
$$

then the $[s d]$ packet process at link $i j$ at time $t$ is described by

$$
\dot{N}_{i j[s d]}(t)=r_{[s d]}(t) * V_{i j[s d]}(t)-\mu_{i j}(t) * \rho_{i j[s d]}\left(\mathbf{N}_{i j}(t)\right) \quad \forall i j \quad \forall[s d]
$$

The state of the network is described by the expected number of virtual circuits $V_{i j[s d]}(t)$ and of packets $N_{i j[s d]}(t)$ for each link $i j$ for each $[s d]$ traffic. So, we define the network state as

$$
\mathbf{X}(t)=\left[\begin{array}{c}
\cdots \\
V_{i j[s d]}(t) \\
N_{i j[s d]}(t) \\
\cdots
\end{array}\right]
$$

The control variables are the admission control parameters $\phi_{o[s d]}(t)$ and the routing fractions $\phi_{\pi[s d]}(t)$ for each path $\pi[s d]$, for each $[s d]$ traffic. So, let us define the control vector for the whole network as

$$
\mathbf{U}(t)=\left[\begin{array}{c}
\cdots \\
\phi_{o[s d]}(t) \\
\cdots \\
\phi_{\pi[s d]}(t) \\
\cdots
\end{array}\right]
$$


In order to write the dynamic evolution of the network state in vector form, we define the following auxiliary functions (from equations 1 and 2):

$$
\begin{aligned}
& f_{V, i j[s d]}(t)=\sum_{\pi[s d]} \gamma_{[s d]}(t) * \phi_{\pi[s d]}(t) * 1_{i j \in \pi[s d]}(t)-\delta_{[s d]}(t) * V_{i j[s d]}(t) \quad \forall i j \quad \forall[s d] \\
& f_{N, i j[s d]}(t)=r_{[s d]}(t) * V_{i j[s d]}(t)-\mu_{i j}(t) * \rho_{i j[s d]}\left(\mathbf{N}_{i j}(t)\right) \quad \forall i j \quad \forall[s d] \\
& \mathbf{f}(t, \mathbf{X}(t), \mathbf{U}(t))=\left[\begin{array}{c}
\ldots \\
f_{V, i j[s d]}(t) \\
f_{N, i j[s d]}(t) \\
\cdots
\end{array}\right]
\end{aligned}
$$

Then the network dynamics are described by the following nonlinear differential equation

$$
\dot{\mathbf{X}}(t)=\mathbf{f}(t, \mathbf{X}(t), \mathbf{U}(t))
$$

Thus, we express the network dynamics by a dynamic nonlinear queueing model that takes into consideration the coupling of the virtual circuit and packet processes. In the next section, we use this model to formulate and solve the combined routing and admission control problem for virtual circuit networks as an optimal control problem.

\section{OPTIMAL CONTROL FORMULATION}

First, we define a multi-objective function $g(t, \mathbf{X}(t), \mathbf{U}(t))$ for the joint routing and admission control problem. In datagram networks, one wants to minimize the cost of packet delay, while maximize the revenue of packet throughput. In telephone networks, one wants to minimize the cost of call delay, while maximize the revenue of admitting calls (call throughput). Consequently, in virtual circuit networks, we want to minimize the cost of packet delay and call delay, while maximize the revenue of packet throughput and call throughput. In other words, we want to minimize the lost profit (revenue) of rejecting virtual circuits from the network, of maintaining the virtual circuits and packets inside the network, while maximimize the profit (revenue) from servicing packets. To give the network administrator more flexibility for operating the network, we define the following nonnegative costs and profits (for example in money), at time $t$ : 
$C_{o[s d]}(t) \quad$ : profit from admitting a new $[s d]$ virtual circuit into the network,

$C_{V, i j[s d]}(t) \quad:$ cost/time-unit for an $[s d]$ virtual circuit at link $i j$, for example the cost for maintaining the virtual circuit path through link $i j$, for a time-unit,

$C_{N, i j[s d]}(t) \quad:$ cost/time-unit for an $[s d]$ packet at link $i j$,

$C_{\mu, i j[s d]}(t) \quad$ : profit from servicing an $[s d]$ packet at link $i j$.

Real-life examples of these costs are:

$C_{o[s d]}$ : charge paid for establishing a new $[s d]$ virtual circuit,

$C_{V, i j[s d]}$ : cost paid during a time-unit for reserving resources (eg. buffers, identification numbers, etc.) for open $[s d]$ virtual circuits, for having the processor looking for packets from open $[s d]$ virtual circuits etc.

$C_{N, i j[s d]}$ : fine paid for a unit delay for an $[s d]$ packet,

$C_{\mu, i j[s d]}$ : profit from charge paid for servicing an $[s d]$ packet.

Note also, that these costs/profits are chosen such that the four terms of the objective function have the same dimension (eg. money). Furthermore, they may depend on time (eg. reduced tariff costs during night). Similar costs are also used in $[13,14]$ for telephone and datagram networks.

So, given an initial time $t_{0}$ and a final time $t_{f}$, we define the following time-dependent multi-objective function of the state $\mathbf{X}(t)$ and the controls $\mathbf{U}(t)$ :

$$
\begin{aligned}
g(t, \mathbf{X}(t), \mathbf{U}(t))= & \sum_{[s d]} C_{o[s d]}(t) * \gamma_{[s d]}(t) * \phi_{o[s d]}(t) \\
& +\sum_{[s d]} \sum_{i j} C_{V, i j[s d]}(t) * V_{i j[s d]}(t) \\
& +\sum_{[s d]} \sum_{i j} C_{N, i j[s d]}(t) * N_{i j[s d]}(t) \\
& -\sum_{[s d]} \sum_{i j} C_{\mu, i j[s d]}(t) * \mu_{i j}(t) * \rho_{i j[s d]}\left(\mathbf{N}_{i j}(t)\right)
\end{aligned}
$$

The first term of the objective function is the average loss of not admitting new virtual 
circuits into the network. The second term is the average cost/time-unit for maintaining virtual circuits. The third term is the average cost/time-unit for having packets into the network. Finally, the last term is the profit from servicing packets.

Next, we define the set for the controls as

$$
\begin{array}{cl}
\boldsymbol{\Phi}=\{ & \phi_{o[s d]}(t), \phi_{\pi[s d]}(t) \forall \pi[s d] \quad \forall[s d], \quad \text { such that } \\
& \phi_{o[s d]}(t) \geq 0, \quad \phi_{\pi[s d]}(t) \geq 0 \quad \forall \pi[s d] \quad \forall[s d], \\
& \left.\phi_{o[s d]}(t)+\sum_{\pi[s d]} \phi_{\pi[s d]}(t)=1 \quad \forall[s d]\right\}
\end{array}
$$

Nonnegative constraints on the network state $V_{i j[s d]}(t) \geq 0$ and $N_{i j[s d]}(t) \geq 0$ are always satisfied due to the structure of $\mathbf{f}(t, \mathbf{X}(t), \mathbf{U}(t))$.

Define also $P_{V, i j[s d]}(t)$ to be the costate variable for $V_{i j[s d]}(t)$, the expected number of $[s d]$ virtual circuits on link $i j$, and $P_{N, i j[s d]}(t)$ to be the costate variable for $N_{i j[s d]}(t)$, the expected number of $[s d]$ packets on link $i j$. Then the costate variable vector for all links $i j$ for all $[s d]$ processes is $\mathbf{P}(t)=\left[\ldots P_{V, i j[s d]}(t) P_{N, i j[s d]}(t) \ldots\right]^{T}$.

Then our Dynamic Virtual Circuit Routing and Admission Control problem (DVCRAC) is [9]:

Problem DVCRAC:

$$
\begin{array}{ll}
\text { minimize } & \int_{t_{0}}^{t_{f}} g(t, \mathbf{X}(t), \mathbf{U}(t)) d t \\
\text { with respect to } & \mathbf{U}(t) \\
\text { such that } & \dot{\mathbf{X}}(t)=\mathbf{f}(t, \mathbf{X}(t), \mathbf{U}(t)) \\
& \mathbf{X}\left(t_{0}\right)=\mathbf{X}_{0} \\
& \mathbf{X}\left(t_{f}\right) \text { free } \\
& \mathbf{U}(t) \in \mathbf{\Phi}
\end{array}
$$


where

$\begin{array}{ll}t_{0} & \text { fixed initial time, } \\ t_{f} & \text { fixed final time, } \\ \mathbf{X}(t) & \text { network state, } \\ \mathbf{U}(t) & \text { controls, } \\ g(t, \mathbf{X}(t), \mathbf{U}(t)) & \text { objective function, } \\ \mathbf{f}(t, \mathbf{X}(t), \mathbf{U}(t)) & \text { state dynamics, } \\ \mathbf{\Phi} & \text { control set, } \\ \mathbf{X}\left(t_{0}\right)=\mathbf{X}_{0} & \text { initial network state, } \\ \mathbf{X}\left(t_{f}\right) & \text { final network state, }\end{array}$

The Hamiltonian function of the state $\mathbf{X}(t)$, the controls $\mathbf{U}(t)$ and the costate variables $\mathbf{P}(t)$ at time $t$ is

$$
H(t, \mathbf{X}(t), \mathbf{U}(t), \mathbf{P}(t))=g(t, \mathbf{X}(t), \mathbf{U}(t))+\mathbf{P}(t) * \mathbf{f}(t, \mathbf{X}(t), \mathbf{U}(t))
$$

Note that the objective function $g$ in the Hamiltonian has a multiplier equal to 1 , since we have free final state conditions.

Next, we provide necessary and sufficient conditions for optimality.

Theorem 1. Necessary conditions

Let $\mathbf{U}^{*}(t)$ be a piecewise continous control defined on $\left[t_{0}, t_{f}\right]$ which solves Problem DVCRAC and let $\mathbf{X}^{*}(t)$ be the associated optimal path. Then there exists a continuous and piecewise continuously differentiable vector function $\mathbf{P}(t)=\left[\ldots P_{V, i j[s d]}(t) P_{N, i j[s d]}(t) \ldots\right]^{T}$ such that the following conditions are satisfied for all $t \in\left[t_{0}, t_{f}\right]$,

$$
\begin{aligned}
& \phi_{o[s d]}^{*}(t) \begin{cases}>0 & \text { only if } C_{o[s d]}(t)=\min \left\{C_{o[s d]}(t), \min _{p[s d]}\left\{\sum_{i j} P_{V, i j[s d]}(t) * 1_{i j \in p[s d]}(t)\right\}\right\} \\
=0 & \text { o.w. } \forall[s d]\end{cases} \\
& \phi_{\pi[s d]}^{*}(t)\left\{\begin{aligned}
>0 \quad \text { only } & \text { if } \sum_{i j} P_{V, i j[s d]}(t) * 1_{i j \in \pi[s d]}(t)= \\
& =\min \left\{C_{o[s d]}(t), \min _{p[s d]}\left\{\sum_{i j} P_{V, i j[s d]}(t) * 1_{i j \in p[s d]}(t)\right\}\right\} \\
=0 & \text { o.w. } \forall \pi[s d] \quad \forall[s d]
\end{aligned}\right.
\end{aligned}
$$




$$
\begin{aligned}
& \dot{V}_{i j[s d]}^{*}(t) \quad=\sum_{\pi[s d]} \gamma_{[s d]}(t) * \phi_{\pi[s d]}^{*}(t) * 1_{i j \in \pi[s d]}(t)-\delta_{[s d]}(t) * V_{i j[s d]}^{*}(t) \quad \forall i j \quad \forall[s d] \\
& \dot{N}_{i j[s d]}^{*}(t) \quad=\quad r_{[s d]}(t) * V_{i j[s d]}^{*}(t)-\mu_{i j}(t) * \rho_{i j[s d]}\left(\mathbf{N}_{i j}^{*}(t)\right) \quad \forall i j \quad \forall[s d] \\
& V_{i j[s d]}^{*}\left(t_{0}\right)=V_{i j[s d], 0} \quad \forall i j \quad \forall[s d] \\
& N_{i j[s d]}^{*}\left(t_{0}\right)=N_{i j[s d], 0} \quad \forall i j \quad \forall[s d] \\
& \dot{P}_{V, i j[s d]}(t) \quad=-\left(C_{V, i j[s d]}(t)-P_{V, i j[s d]}(t) * \delta_{[s d]}(t)+P_{N, i j[s d]}(t) * r_{[s d]}(t)\right) \forall i j \quad \forall[s d] \\
& \dot{P}_{N, i j[s d]}(t)=-\left(C_{N, i j[s d]}(t)-\sum_{\left[s_{1} d_{1}\right]} C_{\mu, i j\left[s_{1} d_{1}\right]}(t) * \mu_{i j}(t) * \frac{d \rho_{i j\left[s_{1} d_{1}\right]}\left(\mathbf{N}_{i j}^{*}(t)\right)}{d N_{i j[s d]}(t)}-\right. \\
& \left.-\sum_{\left[s_{1} d_{1}\right]} P_{N, i j\left[s_{1} d_{1}\right]}(t) * \mu_{i j}(t) * \frac{d \rho_{i j\left[s_{1} d_{1}\right]}\left(\mathbf{N}_{i j}^{*}(t)\right)}{d N_{i j[s d]}(t)}\right) \quad \forall i j \quad \forall[s d] \\
& P_{V, i j[s d]}\left(t_{f}\right)=0 \quad \forall i j \quad \forall[s d] \\
& P_{N, i j[s d]}\left(t_{f}\right)=0 \quad \forall i j \quad \forall[s d]
\end{aligned}
$$

Proof: see Appendix A.

\section{Theorem 2. Sufficient conditions}

Let $(\overline{\mathbf{X}}(t), \overline{\mathbf{U}}(t))$ be an admissible pair in Problem DVCRAC. Assume that $\rho_{i j[s d]}\left(\mathbf{N}_{i j}(t)\right)$ is defined for $\mathbf{N}_{i j}(t) \geq 0$, is concave monotonically increasing and twice differentiable in $\mathbf{N}_{i j}(t)$. If there exists a continuous and piecewise continuously differentiable vector function $\mathbf{P}(t)=\left[\ldots P_{V, i j[s d]}(t) P_{N, i j[s d]}(t) \ldots\right]^{T}$ such that the following conditions are satisfied for all $t \in\left[t_{0}, t_{f}\right]$

$$
\bar{\phi}_{o[s d]}(t) \begin{cases}>0 & \text { only if } C_{o[s d]}(t)=\min \left\{C_{o[s d]}(t), \min _{p[s d]}\left\{\sum_{i j} P_{V, i j[s d]}(t) * 1_{i j \in p[s d]}(t)\right\}\right\} \\ =0 & \text { o.w. } \forall[s d]\end{cases}
$$




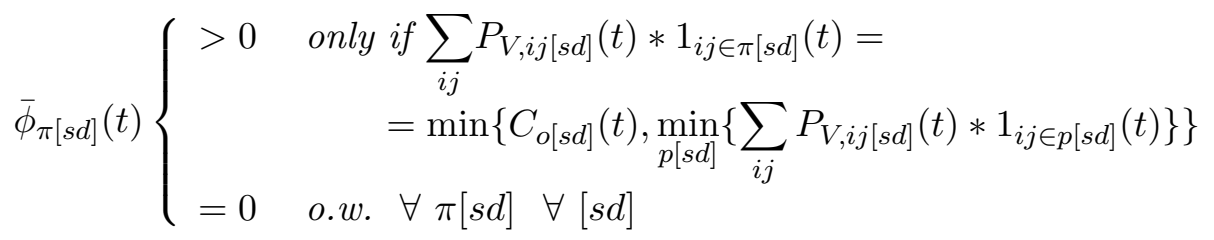

$$
\begin{aligned}
& \dot{\bar{V}}_{i j[s d]}(t) \quad=\sum_{\pi[s d]} \gamma_{[s d]}(t) * \bar{\phi}_{\pi[s d]}(t) * 1_{i j \in \pi[s d]}(t)-\delta_{[s d]}(t) * \bar{V}_{i j[s d]}(t) \quad \forall i j \quad \forall[s d] \\
& \dot{\bar{N}}_{i j[s d]}(t) \quad=r_{[s d]}(t) * \bar{V}_{i j[s d]}(t)-\mu_{i j}(t) * \rho_{i j[s d]}\left(\overline{\mathbf{N}}_{i j}(t)\right) \quad \forall i j \quad \forall[s d] \\
& \bar{V}_{i j[s d]}\left(t_{0}\right)=V_{i j[s d], 0} \quad \forall i j \quad \forall[s d] \\
& \bar{N}_{i j[s d]}\left(t_{0}\right)=N_{i j[s d], 0} \quad \forall i j \quad \forall[s d] \\
& \dot{P}_{V, i j[s d]}(t) \quad=-\left(C_{V, i j[s d]}(t)-P_{V, i j[s d]}(t) * \delta_{[s d]}(t)+P_{N, i j[s d]}(t) * r_{[s d]}(t)\right) \forall i j \quad \forall[s d] \\
& \dot{P}_{N, i j[s d]}(t)=\left(C_{N, i j[s d]}(t)-\sum_{\left[s_{1} d_{1}\right]} C_{\mu, i j\left[s_{1} d_{1}\right]}(t) * \mu_{i j}(t) * \frac{d \rho_{i j\left[s_{1} d_{1}\right]}\left(\overline{\mathbf{N}}_{i j}(t)\right)}{d N_{i j[s d]}(t)}-\right. \\
& \left.-\sum_{\left[s_{1} d_{1}\right]} P_{N, i j\left[s_{1} d_{1}\right]}(t) * \mu_{i j}(t) * \frac{d \rho_{i j\left[s_{1} d_{1}\right]}\left(\overline{\mathbf{N}}_{i j}(t)\right)}{d N_{i j[s d]}(t)}\right) \quad \forall i j \quad \forall[s d] \\
& P_{N, i j[s d]}(t) \quad \geq 0 \quad \forall i j \quad \forall[s d] \\
& P_{V, i j[s d]}\left(t_{f}\right)=0 \quad \forall i j \quad \forall[s d] \\
& P_{N, i j[s d]}\left(t_{f}\right)=0 \quad \forall i j \quad \forall[s d]
\end{aligned}
$$

Proof: see Appendix B.

So, after numerically solving a two-Point Boundary-Value Problem (TPBVP), we have the optimal admission control and routing decisions. Numerical methods [15, 16] for the solution of such problems involve either flooding or iterative procedures. Flooding (or dy- 
namic programming) procedures start from a point that satisfies one boundary condition and generates a trajectory. This is repeated many times until one of these trajectories satisfies the other condition or an interpolation of these trajectories can give an acceptable solution. Iterative procedures use successive linearization. A nominal solution is chosen such that to satisfy one or more of the following conditions: 1) state differential equations, 2 ) adjoint differential equations, 3) optimality conditions, 4) boundary conditions. Then this nominal solution is modified by successive linearization such that the remaining conditions are also satisfied. Three classes of iterative procedures may be used: i) neighboring extremal, ii) gradient, and iii) quasi-linearization procedures.

In this paper, we are primarily interested in the optimal control formulation for the infinite horizon problem and the long-run stationary equilibrium solution. So, we will not discuss further numerical techniques for the finite horizon optimal control problem.

In this section, we formulate the combined dynamic routing and admission control problem for virtual circuit networks as an optimal control problem. Then for specific network configuration and traffic characteristics, we can find the optimum admission control and routing decisions by solving a TPBVP. We can decompose the above problem to many smaller subproblems, one for every source-destination. However, numerical solution may require long computational times for on line implementation. Therefore, in the next section, we also derive state-dependent routing and admission controls for the long-run stationary equilibrium that can be used for on-line implementation.

\section{STATE-DEPENDENT ROUTING \& ADMISSION CONTROLS}

In this section, we consider a network with constant arrival rates and mean durations of virtual circuits, as well as constant costs and profits (autonomous system), and we find optimal state-dependent virtual circuit routing and admission controls for the long-run stationary equilibrium.

First, we find an expression for the function $\rho_{i j[s d]}\left(\mathbf{N}_{i j}\right)$. For an $M / M / 1$ or Processor Sharing queueing model the expected number of $[s d]$ packets on link $i j$ at steady state is given by

$$
N_{i j[s d]}=\frac{\rho_{i j[s d]}}{1-\sum_{\left[s_{1} d_{1}\right]} \rho_{i j\left[s_{1} d_{1}\right]}} \quad \forall[s d]
$$

Solving the above system of equations (for all $[s d]$ traffic that use link $i j$ ), we have the 
utilization of link $i j$ for each $[s d]$ process at steady state

$$
\rho_{i j[s d]}=\frac{N_{i j[s d]}}{1+\sum_{\left[s_{1} d_{1}\right]} N_{i j\left[s_{1} d_{1}\right]}}
$$

This function is defined for $\mathbf{N}_{i j} \geq 0$, is concave, monotonically increasing and twice differentiable in $\mathbf{N}_{i j}$ with $\lim _{\mathbf{N}_{i j} \rightarrow \infty} \rho_{i j}\left(\mathbf{N}_{i j}\right)=1$.

Next, we evaluate the costate variables $P_{N, i j[s d]}$ (for the expected number of $[s d]$ packets) and $P_{V, i j[s d]}$ (for the expected number of $[s d]$ virtual circuits).

Lemma 1. For the long-run stationary equilibrium of the virtual circuit routing and admission control problem, the costate variables for the expected number of packets are given by:

$$
\begin{aligned}
P_{N, i j[s d]}=\frac{1+\sum_{\left[s_{1} d_{1}\right]} N_{i j\left[s_{1} d_{1}\right]}^{*}}{\mu_{i j}} * & \left(C_{N, i j[s d]} *\left(1+N_{i j[s d]}^{*}\right)+\right. \\
& \left.+\sum_{\left[s_{2} d_{2}\right] \neq[s d]} C_{N, i j\left[s_{2} d_{2}\right]} * N_{i j\left[s_{2} d_{2}\right]}^{*}\right)-C_{\mu, i j[s d]} \forall i j \forall[s d]
\end{aligned}
$$

Proof: see Appendix C.

In Theorem 2, the condition $P_{N, i j[s d]} \geq 0$ must hold:

$$
\frac{C_{N, i j[s d]}}{\mu_{i j}}-C_{\mu, i j[s d]} \geq 0 \quad \forall[s d]
$$

i.e. the cost should be greater or equal to the profit from servicing a packet (in order to have meaning the $D V C R A C$ problem).

Lemma 2. For the long-run stationary equilibrium of the virtual circuit routing and admission control problem, the costate variables for the expected number of virtual circuits are given by: 


$$
\begin{aligned}
P_{V, i j[s d]}=\frac{C_{V, i j[s d]}}{\delta_{[s d]}}+\frac{r_{[s d]}}{\delta_{[s d]}} * & {\left[\frac { 1 + \sum _ { [ s _ { 1 } d _ { 1 } ] } N _ { i j [ s _ { 1 } d _ { 1 } ] } ^ { * } } { \mu _ { i j } } * \left(C_{N, i j[s d]} *\left(1+N_{i j[s d]}^{*}\right)+\right.\right.} \\
& \left.\left.+\sum_{\left[s_{2} d_{2}\right] \neq[s d]} C_{N, i j\left[s_{2} d_{2}\right]} * N_{i j\left[s_{2} d_{2}\right]}^{*}\right)-C_{\mu, i j[s d]]}\right] \forall i j \forall[s d]
\end{aligned}
$$

Proof: see Appendix D.

Now, we give the state-dependent routing and admission controls.

\section{Theorem 3. Admission Control}

For the long-run stationary equilibrium of the virtual circuit routing and admission control problem, at every source node $s$, for every destination node $d,[s d]$ virtual circuits are rejected at node $s$ only if the cost of rejecting them is less than the minimum cost to go from node $s$ to the destination node $d$ through any of the paths $\pi[s d]$ :

$$
\begin{aligned}
& \phi_{o[s d]}^{*}>0 \text { only if } \\
& C_{o[s d]}<\min _{p[s d]}\left\{\sum _ { i j \in p [ s d ] } \left\{\frac{C_{V, i j[s d]}}{\delta_{[s d]}}+\frac{r_{[s d]}}{\delta_{[s d]}} *\left[\frac { 1 + \sum _ { [ s _ { 1 } d _ { 1 } ] } N _ { i j [ s _ { 1 } d _ { 1 } ] } ^ { * } } { \mu _ { i j } } * \left(C_{N, i j[s d]} *\left(1+N_{i j[s d]}^{*}\right)+\right.\right.\right.\right. \\
&\left.\left.\left.\left.+\sum_{\left[s_{2} d_{2}\right] \neq[s d]} C_{N, i j\left[s_{2} d_{2}\right]} * N_{i j\left[s_{2} d_{2}\right]}^{*}\right)-C_{\mu, i j[s d]}\right]\right\}\right\}
\end{aligned}
$$

Proof: see Appendix E.

\section{Theorem 4. Routing Rule}

For the long-run stationary equilibrium of the virtual circuit routing and admission control problem, $[s d]$ virtual circuits are routed through path $\pi[s d]$ only if the minimum cost to reach the destination node d through path $\pi[s d]$ is the minimum: 


$$
\begin{aligned}
& \phi_{\pi[s d]}^{*}>0 \text { only if } \\
& \sum_{i j \in \pi[s d]}\left\{\frac{C_{V, i j[s d]}}{\delta_{[s d]}}+\frac{r_{[s d]}}{\delta_{[s d]}} *\left[\frac { 1 + \sum _ { [ s _ { 1 } d _ { 1 } ] } N _ { i j [ s _ { 1 } d _ { 1 } ] } ^ { * } } { \mu _ { i j } } * \left(C_{N, i j[s d]} *\left(1+N_{i j[s d]}^{*}\right)+\right.\right.\right. \\
& \left.\left.\left.+\sum_{\left[s_{2} d_{2}\right] \neq[s d]} C_{N, i j\left[s_{2} d_{2}\right]} * N_{i j\left[s_{2} d_{2}\right]}^{*}\right)-C_{\mu, i j[s d]}\right]\right\}= \\
& =\min _{p[s d]}\left\{C_{o[s d]}, \sum_{i j \in p[s d]}\left\{\frac{C_{V, i j[s d]}}{\delta_{[s d]}}+\frac{r_{[s d]}}{\delta_{[s d]}} *\left[\begin{array} { l } 
{ 1 + \sum _ { [ s _ { 1 } d _ { 1 } ] } N _ { i j [ s _ { 1 } d _ { 1 } ] } ^ { * } } \\
{ \mu _ { i j } }
\end{array} \left(C_{N, i j[s d]} *\left(1+N_{i j[s d]}^{*}\right)+\right.\right.\right.\right. \\
& \left.\left.\left.\left.+\sum_{\left[s_{2} d_{2}\right] \neq[s d]} C_{N, i j\left[s_{2} d_{2}\right]} * N_{i j\left[s_{2} d_{2}\right]}^{*}\right)-C_{\mu, i j[s d]}\right]\right\}\right\}
\end{aligned}
$$

Proof: see Appendix E.

From the above analysis, we derive that the length "seen" by an $[s d]$ virtual circuit on link $i j$ is

$$
\begin{gathered}
l_{i j[s d]}=\frac{C_{V, i j[s d]}}{\delta_{[s d]}}+\frac{r_{[s d]}}{\delta_{[s d]}} *\left[\frac { 1 + \sum _ { [ s _ { 1 } d _ { 1 } ] } N _ { i j [ s _ { 1 } d _ { 1 } ] } ^ { * } } { \mu _ { i j } } * \left(C_{N, i j[s d]} *\left(1+N_{i j[s d]}^{*}\right)+\right.\right. \\
\left.\left.+\sum_{\left[s_{2} d_{2}\right] \neq[s d]} C_{N, i j\left[s_{2} d_{2}\right]} * N_{i j\left[s_{2} d_{2}\right]}^{*}\right)-C_{\mu, i j[s d]}\right]
\end{gathered}
$$

The first term of this link length represents $C_{V, i j[s d]}$ (the cost/time-unit for maintaining an $[s d]$ virtual circuit passing through link $i j$ ) times $1 / \delta_{[s d]}$ (the average virtual circuit duration). The second term represents $r_{[s d]} / \delta_{[s d]}$ (the average number of packets in the 
$[s d]$ virtual circuit) times a function for an arbitrary $[s d]$ packet on link $i j$. The first term of this function is $C_{N, i j[s d]}$ (the cost/time-unit for this packet) times the marginal packet delay. The second term of this function is $C_{\mu, i j[s d]}$ (the profit from servicing the packet).

Thus, we express the long-run stationary routing and admission controls as functions of the long-run average network state. For on-line implementation, we repeatedly measure the instantaneous network state over a time interval and approximate the average network state using these measurements. We use this average network state to calculate the lengths "seen" by a newly arriving virtual circuit. When a new virtual circuit arrives to the network, we reject or route it to its destination via the minimum length path.

In [10], we consider the special case where we have zero virtual circuit maintenance $\operatorname{cost} C_{V, i j[s d]}=0$, zero profit $C_{\mu, i j[s d]}=0$ for servicing packets, and unit delay costs $C_{N, i j\left[s_{2} d_{2}\right]}=1, \forall\left[s_{2} d_{2}\right]$ on link $i j$. Then the length of link $i j$ for an [sd] virtual circuit is

$$
l_{i j[s d]}=\frac{r_{[s d]}}{\delta_{[s d]}} * \frac{\left(1+\sum_{\left[s_{1} d_{1}\right]} N_{i j\left[s_{1} d_{1}\right]}^{*}\right)^{2}}{\mu_{i j}}
$$

So, when the only objective to minimize is the average packet delay, then the link length is given by a quadratic function of the average number of packets on this link.

We show via simulation, that this quadratic routing is better than the shortest-queue routing. Also, the sooner the network state information becomes available to the router and the more often that this information is updated, the smaller the achieved average packet delay. Also, the age of this information at the router should be less or at least comparable to (but not extremely larger than) the mean interarrival time of virtual circuits.

\section{CONCLUSIONS}

In this paper, we present nonlinear dynamic queueing models of multiple class multiple source-destination virtual circuit networks, by explicitly considering the interaction among the virtual circuit and the packet processes.

We formulate the dynamic virtual circuit routing and admission control problem as an optimal control problem. We set up a multi-objective function and solve it using Pontryagin's maximum principle.

Then we derive state-dependent routing and admission control policies for virtual circuit network control. Finally, we define as link length a function of the mean virtual circuit 
duration, the mean number of packets generated in each virtual circuit, and the average number of packets on this link.

Future research may be directed towards the modeling area and the performance objective area. Proposals for future high speed networks consider more than two levels for the transport mode, for example: virtual call level, burst (or talk/silence) level, and packet (or cell) level. Our analysis may be generalized to more than two levels of processes. Another modeling development is to consider more complicated processes (general distributions, batches, complex service scheduling, limited buffers etc.). Then, new dynamic models should be derived to describe these processes. Finally, other performance objectives may also be considered, such as bounded packet loss probability, bounded packet delay, jitter of the delay etc.

\section{APPENDIX A}

Proof: Necessary conditions for optimality are provided by Pontryagin's maximum principle $[15,17]$. The Hamiltonian must satisfy the following condition

$$
H\left(t, \mathbf{X}^{*}(t), \mathbf{U}^{*}(t), \mathbf{P}(t)\right) \leq H\left(t, \mathbf{X}^{*}(t), \mathbf{U}, \mathbf{P}(t)\right) \quad \forall \mathbf{U} \in \mathbf{\Phi}
$$

which is equivalent to the following condition

$$
\begin{aligned}
& \sum_{[s d]}\left\{\gamma_{[s d]}(t) *\left[C_{o[s d]}(t) * \phi_{o[s d]}^{*}(t)+\sum_{\pi[s d]} \sum_{i j} P_{V, i j[s d]}(t) * \phi_{\pi[s d]}^{*}(t) * 1_{i j \in \pi[s d]}(t)\right]\right\} \leq \\
& \leq \sum_{[s d]}\left\{\gamma_{[s d]}(t) *\left[C_{o[s d]}(t) * \phi_{o[s d]}(t)+\sum_{\pi[s d]} \sum_{i j} P_{V, i j[s d]}(t) * \phi_{\pi[s d]}(t) * 1_{i j \in \pi[s d]}(t)\right]\right\} \\
& \forall \phi_{o[s d]}, \phi_{\pi[s d]} \in \mathbf{\Phi} \quad \forall \pi[s d] \quad \forall[s d]
\end{aligned}
$$

Since, there is no dependency among the controls for different source-destination pairs $[s d]$, we can decomposed the above conditions $\forall[s d]$ to

$$
\begin{aligned}
& \gamma_{[s d]}(t) *\left[C_{o[s d]}(t) * \phi_{o[s d]}^{*}(t)+\sum_{\pi[s d]} \sum_{i j} P_{V, i j[s d]}(t) * \phi_{\pi[s d]}^{*}(t) * 1_{i j \in \pi[s d]}(t)\right] \leq \\
& \leq \gamma_{[s d]}(t) *\left[C_{o[s d]}(t) * \phi_{o[s d]}(t)+\sum_{\pi[s d]} \sum_{i j} P_{V, i j[s d]}(t) * \phi_{\pi[s d]}(t) * 1_{i j \in \pi[s d]}(t)\right] \\
& \forall \phi_{o[s d]}, \phi_{\pi[s d]} \in \mathbf{\Phi} \quad \forall \pi[s d]
\end{aligned}
$$


Then the optimal controls satisfy the following conditions

$$
\begin{aligned}
& \phi_{o[s d]}^{*}(t)\left\{\begin{array}{cc}
>0 & \text { only if } C_{o[s d]}(t)=\min \left\{C_{o[s d]}(t), \min _{p[s d]}\left\{\sum_{i j} P_{V, i j[s d]}(t) * 1_{i j \in p[s d]}(t)\right\}\right\} \\
=0 & \text { o.w. } \forall[s d]
\end{array}\right. \\
& \phi_{\pi[s d]}^{*}(t)\left\{\begin{array}{cc}
>0 & \text { only if } \sum_{i j} P_{V, i j[s d]}(t) * 1_{i j \in \pi[s d]}(t)= \\
\min \left\{C_{o[s d]}(t), \min _{p[s d]}\left\{\sum_{i j} P_{V, i j[s d]}(t) * 1_{i j \in p[s d]}(t)\right\}\right\} \\
=0 & \text { o.w. } \forall \pi[s d] \quad \forall[s d]
\end{array}\right.
\end{aligned}
$$

The optimal state and control pair $\left(\mathbf{X}^{*}(t), \mathbf{U}^{*}(t)\right)$ must also satisfy the state dynamics $\dot{\mathbf{X}}^{*}(t)=\mathbf{f}\left(t, \mathbf{X}^{*}(t), \mathbf{U}^{*}(t)\right)$

which can be rewritten as

$$
\begin{aligned}
& \dot{V}_{i j[s d]}^{*}(t)=\sum_{\pi[s d]} \gamma_{[s d]}(t) * \phi_{\pi[s d]}^{*}(t) * 1_{i j \in \pi[s d]}(t)-\delta_{[s d]}(t) * V_{i j[s d]}^{*}(t) \quad \forall i j \quad \forall[s d] \\
& \dot{N}_{i j[s d]}^{*}(t)=r_{[s d]}(t) * V_{i j[s d]}^{*}(t)-\mu_{i j}(t) * \rho_{i j[s d]}\left(\mathbf{N}_{i j}^{*}(t)\right) \quad \forall i j \quad \forall[s d]
\end{aligned}
$$

The optimal state must also satisfy the initial state $\mathbf{X}^{*}\left(t_{0}\right)=\mathbf{X}_{0}$, therefore

$$
\begin{array}{llll}
V_{i j[s d]}^{*}\left(t_{0}\right) & =V_{i j[s d], 0} & \forall i j & \forall[s d] \\
N_{i j[s d]}^{*}\left(t_{0}\right)=N_{i j[s d], 0} & \forall i j & \forall[s d]
\end{array}
$$

The costate variables must satisfy the following conditions

$\dot{\mathbf{P}}(t)=-\nabla_{\mathbf{X}} H\left(t, \mathbf{X}^{*}(t), \mathbf{U}^{*}(t), \mathbf{P}(t)\right)$

which can be rewritten as 


$$
\begin{aligned}
& \dot{P}_{V, i j[s d]}(t)=-\frac{\partial H\left(t, \mathbf{X}^{*}(t), \mathbf{U}^{*}(t), \mathbf{P}(t)\right)}{\partial V_{i j[s d]}(t)}= \\
&=-\left\{C_{V, i j[s d]}(t)-P_{V, i j[s d]}(t) * \delta_{[s d]}(t)+P_{N, i j[s d]}(t) * r_{[s d]}(t)\right\} \quad \forall i j \quad \forall[s d] \\
& \dot{P}_{N, i j[s d]}(t)=-\frac{\partial H\left(t, \mathbf{X}^{*}(t), \mathbf{U}^{*}(t), \mathbf{P}(t)\right)}{\partial N_{i j[s d]}(t)}= \\
&=-\left\{C_{N, i j[s d]}(t)-\sum_{\left[s_{1} d_{1}\right]} C_{\mu, i j\left[s_{1} d_{1}\right]}(t) * \mu_{i j}(t) * \frac{d \rho_{i j\left[s_{1} d_{1}\right]}\left(\mathbf{N}_{i j}^{*}(t)\right)}{d N_{i j[s d]}(t)}-\right. \\
& \\
&\left.\quad-\sum_{\left[s_{1} d_{1}\right]} P_{N, i j\left[s_{1} d_{1}\right]}(t) * \mu_{i j}(t) * \frac{d \rho_{i j\left[s_{1} d_{1}\right]}\left(\mathbf{N}_{i j}^{*}(t)\right)}{d N_{i j[s d]}(t)}\right\} \quad \forall i j \quad \forall[s d]
\end{aligned}
$$

Since we have no conditions on the final state $\mathbf{X}\left(t_{f}\right)$, the costate variables at the final time must be zero, $\mathbf{P}\left(t_{f}\right)=0$. Therefore

$$
\begin{array}{lll}
P_{V, i j[s d]}\left(t_{f}\right)=0 & \forall i j & \forall[s d] \\
P_{N, i j[s d]}\left(t_{f}\right)=0 & \forall i j & \forall[s d]
\end{array}
$$

\section{APPENDIX B}

Proof: The first part of the proof is similar to that of Theorem 1.

In addition, the control set $\boldsymbol{\Phi}$ is a convex set and since $-\rho_{i j[s d]}\left(\mathbf{N}_{i j}(t)\right)$ is a convex (i.e. $\rho_{i j[s d]}\left(\mathbf{N}_{i j}(t)\right)$ is concave) and differentiable function in $\mathbf{N}_{i j}(t)$, our objective function $g(t, \mathbf{X}(t), \mathbf{U}(t))$, as well as each component of $\mathbf{f}(t, \mathbf{X}(t), \mathbf{U}(t))$ are differentiable and convex functions in the variables $(\mathbf{X}(t), \mathbf{U}(t))$ for $t \in\left[t_{0}, t_{f}\right]$. Furthermore, if $P_{N, i j[s d]}(t) \geq$ $0 \quad \forall$ ij $\forall[s d]$, then the Hamiltonian function $H(t, \mathbf{X}(t), \mathbf{U}(t), \mathbf{P}(t))$ is a convex function in $(\mathbf{X}(t), \mathbf{U}(t))$ for $t \in\left[t_{0}, t_{f}\right]$ (we need nonnegativity of the costate variables only for those components of $\mathbf{f}(t, \mathbf{X}(t), \mathbf{U}(t))$ that are nonlinear in $\mathbf{X}(t)[18,19])$.

If all the above conditions are satisfied, then $(\overline{\mathbf{X}}(t), \overline{\mathbf{U}}(t))$ is optimal.

\section{APPENDIX C}


Proof: At steady state, the costate variables must satisfy $\dot{P}_{N, i j[s d]}=0 \quad \forall i j \quad \forall[s d]$. From Theorems 1 and 2:

$$
\begin{aligned}
\dot{P}_{N, i j[s d]}=0 \Rightarrow \quad C_{N, i j[s d]}-\sum_{\left[s_{1} d_{1}\right]} C_{\mu, i j\left[s_{1} d_{1}\right]} * \mu_{i j} * \frac{d \rho_{i j\left[s_{1} d_{1}\right]}\left(\mathbf{N}_{i j}^{*}\right)}{d N_{i j[s d]}}- \\
\quad-\sum_{\left[s_{1} d_{1}\right]} P_{N, i j\left[s_{1} d_{1}\right]} * \mu_{i j} * \frac{d \rho_{i j\left[s_{1} d_{1}\right]}\left(\mathbf{N}_{i j}^{*}\right)}{d N_{i j[s d]}}=0 \quad \forall i j \quad \forall[s d]
\end{aligned}
$$

In order to find the costate variables $P_{N, i j[s d]}$ (for the expected number of $[s d]$ packets on every link $i j$ ), we must solve a system of equations for all source-destination processes that use this link $i j$ :

$$
\begin{aligned}
& C_{N, i j[s d]}-\sum_{\left[s_{1} d_{1}\right]} C_{\mu, i j\left[s_{1} d_{1}\right]} * \mu_{i j} * \frac{d \rho_{i j\left[s_{1} d_{1}\right]}\left(\mathbf{N}_{i j}^{*}\right)}{d N_{i j[s d]}}- \\
& -\sum_{\left[s_{1} d_{1}\right]} P_{N, i j\left[s_{1} d_{1}\right]} * \mu_{i j} * \frac{d \rho_{i j\left[s_{1} d_{1}\right]}\left(\mathbf{N}_{i j}^{*}\right)}{d N_{i j[s d]}}=0 \quad \forall[s d]
\end{aligned}
$$

Substituting from equation 3 , we rewrite this system of equations of $P_{N, i j[s d]}$, for each link $i j$, as

$$
\begin{aligned}
& C_{N, i j[s d]}-\sum_{\left[s_{1} d_{1}\right]} C_{\mu, i j\left[s_{1} d_{1}\right]} * \mu_{i j} *\left\{\frac{1+\sum_{\left[s_{2} d_{2}\right] \neq[s d]} N_{i j\left[s_{2} d_{2}\right]}^{*}}{\left(1+\sum_{\left[s_{1} d_{1}\right]} N_{i j\left[s_{1} d_{1}\right]}^{*}\right)^{2}}-\sum_{\left[s_{2} d_{2}\right] \neq[s d]} \frac{N_{i j\left[s_{2} d_{2}\right]}^{*}}{\left(1+\sum_{\left[s_{1} d_{1}\right]} N_{i j\left[s_{1} d_{1}\right]}^{*}\right)^{2}}\right\}- \\
& -\sum_{\left[s_{1} d_{1}\right]} P_{N, i j\left[s_{1} d_{1}\right]} * \mu_{i j} *\left\{\frac{1+\sum_{\left[s_{2} d_{2}\right] \neq[s d]} N_{i j\left[s_{2} d_{2}\right]}^{*}}{\left(1+\sum_{\left[s_{1} d_{1}\right]} N_{i j\left[s_{1} d_{1}\right]}^{*}\right)^{2}}-\sum_{\left[s_{2} d_{2}\right] \neq[s d]} \frac{N_{i j\left[s_{2} d_{2}\right]}^{*}}{\left(1+\sum_{\left[s_{1} d_{1}\right]} N_{i j\left[s_{1} d_{1}\right]}^{*}\right)^{2}}\right\}=0 \\
& \forall[s d]
\end{aligned}
$$

The solution to the above system gives the result.

\section{APPENDIX D}


Proof: At steady state, the $P_{V, i j[s d]}$ must satisfy $\dot{P}_{V, i j[s d]}=0 \quad \forall i j \quad \forall[s d]$. From Theorems 1 and 2:

$$
\dot{P}_{V, i j[s d]}=0 \Rightarrow C_{V, i j[s d]}-P_{V, i j[s d]} * \delta_{[s d]}+P_{N, i j[s d]} * r_{[s d]}=0 \quad \forall i j \quad \forall[s d]
$$

Then

$$
P_{V, i j[s d]}=\frac{C_{V, i j[s d]}}{\delta_{[s d]}}+\frac{r_{[s d]}}{\delta_{[s d]}} * P_{N, i j[s d]} \quad \forall i j \quad \forall[s d]
$$

Substituting the $P_{N, i j[s d]}$ (from Lemma 1) into $P_{V, i j[s d]}$ (above) we have the result.

\section{APPENDIX E}

Proof: The minimization of the Hamiltonian with respect to the admission control and routing fractions is equivalent to the following minimization problem

$$
\begin{aligned}
& \text { minimize } \quad \sum_{[s d]}\left\{\gamma_{[s d]} *\left[C_{o[s d]} * \phi_{o[s d]}+\sum_{\pi[s d]} \sum_{i j} P_{V, i j[s d]} * \phi_{\pi[s d]} * 1_{i j \in \pi[s d]}\right]\right\} \\
& \text { with respect to } \quad \phi_{o[s d]}, \phi_{\pi[s d]}, \quad \forall \pi[s d] \quad \forall[s d] \\
& \text { such that } \quad \phi_{o[s d]}+\sum_{\pi[s d]} \phi_{\pi[s d]}=1 \quad \phi_{o[s d]}, \phi_{\pi[s d]} \geq 0 \quad \forall \pi[s d] \quad \forall[s d]
\end{aligned}
$$

where the costate variables $P_{V, i j[s d]}$ (for the expected number of virtual circuits) and $P_{N, i j[s d]}$ (for the expected number of packets) are given by Lemmas 1 and 2.

The above problem can be decomposed for each source-destination pair $[s d]$ to the following problem 
minimize

$$
\gamma_{[s d]} *\left[C_{o[s d]} * \phi_{o[s d]}+\sum_{\pi[s d]} \sum_{i j} P_{V, i j[s d]} * \phi_{i j[s d]} * 1_{i j \in \pi[s d]}\right]
$$

with respect to $\quad \phi_{o[s d]}, \phi_{\pi[s d]} \quad \forall \pi[s d]$

such that

$$
\phi_{o[s d]}+\sum_{\pi[s d]} \phi_{\pi[s d]}=1, \quad \phi_{o[s d]}, \phi_{\pi[s d]} \geq 0 \quad \forall \pi[s d]
$$

Define the minimum cost at source node $s$ for the $[s d]$ virtual circuit traffic to be $P_{V, s[s d]}^{*}=\min \left\{C_{o[s d]}, \min _{p[s d]}\left\{\sum_{i j} P_{V, i j[s d]} * 1_{i j \in p[s d]}\right\}\right\}$. Then the optimum admission controls are:

$$
\phi_{o[s d]}^{*} \begin{cases}>0 & \text { only if } C_{o[s d]}=P_{V, s[s d]}^{*} \\ =0 & \text { o.w. }\end{cases}
$$

and the optimum routing fractions are:

$$
\phi_{\pi[s d]}^{*} \begin{cases}>0 & \text { only if } \sum_{i j} P_{V, i j[s d]} * 1_{i j \in \pi[s d]}=P_{V, s[s d]}^{*} \\ =0 & \text { o.w. }\end{cases}
$$

Therefore, an $[s d]$ virtual circuit is rejected at source node $s$ only if the cost of rejecting it is equal to the minimum cost at node $s$, i.e. $C_{o[s d]}=P_{V, s[s d]}^{*}$. Also, path $\pi[s d]$ will be used for the $[s d]$ traffic only if its costate variable achieves the minimum cost, i.e. $\sum_{i j} P_{V, i j[s d]} * 1_{i j \in \pi[s d]}=P_{V, s[s d]}^{*}$. Substituting from Lemmas 1 and 2 , we have the result.

When the admission control and routing fractions achieve their optimum values, we have

$$
C_{o[s d]} * \phi_{o[s d]}^{*}+\sum_{i j} \sum_{\pi[s d]} P_{V, i j[s d]} * \phi_{i j[s d]}^{*} * 1_{i j \in \pi[s d]}=P_{V, s[s d]}^{*}
$$

\section{ACKNOWLEDGMENTS}


The authors wish to thank Dr. Vikram Saksena of AT \& T Bell Laboratories for suggesting the virtual circuit routing problem and for many useful suggestions. The comments of two anonymous reviewers are gratefully acknowledged,

\section{References}

[1] L. Kleinrock. Queueing Systems, Vol. 1: Theory. J. Wiley \& Sons, 1975.

[2] E.F. Wunderlich. An analysis of dynamic virtual circuit routing. Proc. IEEE National Telecommunications Conference, pp. A3.3. - A3.3.6, IEEE 1981.

[3] A. Gersht. Analytical model of dynamic routing in virtual circuit packet switching network. Proc. International Conference on Computer Communications, pp.76-80, IEEE 1982.

[4] E.M. Gafni and D.P. Bertsekas. Path assignment for virtual circuit routing. Proc. ACM SIGCOMM 1983 Conference, pp. 21-25, ACM 1983.

[5] J.M. Jaffe and A. Segall. Threshold design for dynamic routing. Proc. IEEE International Communications Conference, pp. 90-92, IEEE 1986.

[6] D. Tipper and M.K. Sundareshan. An optimal control approach to decentralized dynamic virtual circuit routing in computer networks. Proc. IEEE Infocom 90, pp. 926-933, IEEE 1990.

[7] A.A. Economides, P.A. Ioannou, and J.A. Silvester. Decentralized adaptive routing for virtual circuit networks using stochastic learning automata. Proc. of IEEE Infocom 88 Conference, pp. 613-622, IEEE 1988.

[8] A.A. Economides, P.A. Ioannou, and J.A. Silvester. Adaptive routing and congestion control for window flow controlled virtual circuit networks. Proc. 27th Annual Allerton Conference on Communications, Control, and Computing, pp. 849-857, Sept. 1989.

[9] A.A. Economides. A unified game-theoretic methodology for the joint load sharing, routing and congestion control problem. Ph.D. Dissertation, University of Southern California, Los Angeles, August 1990.

[10] A. A. Economides, P. A. Ioannou, and J. A. Silvester. Adaptive virtual circuit routing. Computer Networks and ISDN Systems, pp. 1-8, Vol. 28, No 1, 1995. 
[11] M.G.H. Katevenis. Fast switching and fair control of congested flow in broadband networks. IEEE Journal on Selected Areas in Communications, Vol. SAC-5, No. 8, pp. 1315-1326, Oct. 1987.

[12] S.P. Morgan. Window flow control on a trunked bute-stream virtual circuit. IEEE Transactions on Communications, Vol. 36, No. 7, pp. 816-825, July 1988.

[13] J. Filipiak. Optimal control of store-and-forward networks. Optimal Control Applications 83 Methods, Vol. 3, pp. 155-176, 1982.

[14] J. Filipiak. Modelling and Control of Dynamic Flows in Communication Networks. Springer-Verlag, 1988.

[15] A.E.Jr. Bryson and Y. C. Ho. Applied Optimal Control. Hemisphere Publ. Co, 1975.

[16] W.A. Gruver and E. Sachs. Algorithmic Methods in Optimal Control. Pitman Advanced Publ., 1980.

[17] L.D. Berkovitz. Optimal Control Theory. Springer-Verlag, 1974.

[18] M.I. Kamien and N.L. Schwartz. Sufficient conditions in optimal control theory. Journal Economic Theory, Vol. 3, pp. 207-214, 1971.

[19] A. Seierstad and K. Sydsaeter. Sufficient conditions in optimal control theory. International Economic Review, Vol. 18, No. 2, pp. 367-391, June 1977. 